\title{
Teorías del desarrollo sustentable para el siglo XXI: un breve análisis
}

\section{Theories of sustainable development for the twenty-first century: a brief analysis}

\author{
López-González, Álvaro Santigo; Zúniga-González, Carlos Alberto; \\ Angel, Sol-Sánchez; Santivañez-Galarza, José Luis; Editor Academico \\ Dr. Roberto Berrios
}

\author{
Álvaro Santigo López-González \\ alvaro_lopez67@yahoo.es \\ Universidad Nacional Autónoma de Nicaragua. \\ UNAN - Managua., Nicaragua \\ iD Carlos Alberto Zúniga-González \\ czuniga@ct.unanleon.edu.ni \\ Universidad Nacional Autónoma de Nicaragua, León., \\ Nicaragua \\ Dol-Sánchez Angel \\ angel.sols@gmail.com \\ Colegio de Postgraduados México, Nicaragua \\ José Luis Santivañez-Galarza \\ jlsantivanez44@gmail.com \\ CATIE-Bolivia, Nicaragua \\ Editor Academico Dr. Roberto Berrios \\ UNAN Leon Nicaragua, Nicaragua
}

Revista Iberoamericana de Bioeconomía y Cambio Climático

Universidad Nacional Autónoma de Nicaragua, León, Nicaragua ISSN-e: 2410-7980

Periodicidad: Semestral

vol. 2, núm. 1,2016

czuniga@ct.unanleon.edu.ni

Recepción: 15 Febrero 2016

Aprobación: 28 Junio 2016

URL: http://portal.amelica.org/ameli/journal/394/3941750030/

DOI: https://doi.org/10.5377/ribcc.v2i1.5710

Autor de correspondencia: alvaro_lopez67@yahoo.es
Resumen: El presente ensayo se centró en el análisis de las teorías del desarrollo sustentable. Realizamos una revisión de la literatura de los principales trabajos en el tema de la teoría del desarrollo. Los resultados desarrollamos los descriptores de desarrollo sustentable, desarrollo rural, desarrollo ambiental y definimos su enlace multidisciplinar para comprender holísticamente una nueva epistemología del desarrollo. El surgimiento de una propuesta enmarcada en lo que se denomina "Ecología Profunda" es un planteamiento interesante a ser tomado en cuenta por los diseñadores de políticas nacionales, por los teóricos de la economía, por los ambientalistas y por todas las personas relacionadas o no con la imperiosa necesidad del restablecimiento o al menos preservación de las condiciones naturales del mundo externo que nos rodea. Complementa este enfoque la nueva epistemología de la Bioeconomia y el Cambio Climático que aborda los senderos productivo de la bio economía y nuevo instrumento para medir la nueva Economia Bio basada.

Palabras clave: Desarrollo, Ecología, Sustentable, Ambiental, Bioeconomia, Cambio Climático.

Abstract: This essay focused on the analysis of the theories of sustainable development. We carry out a review of the literature of the main works on the subject of development theory. The results we develop the descriptors of sustainable development, rural development, environmental development and define their multidisciplinary link to holistically understand a new epistemology of development. The emergence of a proposal framed in what is called "Deep Ecology" is an interesting approach to be taken into account by designers of national policies, by economic theorists, by environmentalists and by all people related or not with the urgent need to restore or at least preserve the natural conditions of the external world around us. This approach is complemented by the new epistemology of Bioeconomy and Climate Change that addresses the productive paths of the bioeconomy and a new instrument to measure the new Biobased Economy.

Keywords: Devepment, Ecology, Sustainable, Environment, Bioeconomy, Climate Change. 


\section{INTRODUCCIÓN}

El tema del desarrollo sustentable es abordado por los autores (Garza, 2008 ), (Schejtman, 2004), (Altieri, 2000),Artaraz 2002) quienes plantean las principales variables del desarrollo sustentable en la actividad agrícola. Lo interesante de ello es que las variables no son aisladas, sino que de manera, interdisciplinaria se hace la relación del desarrollo con lo económico, ambiental, político, social, etc., para llegar a lo sustentable, así revisar estos planteamientos para concluir en un desarrollo sustentable resulta interesante para los investigadores de este tema.

El escrito es una breve revisión a los diferentes enfoques que en la actualidad se dan al tema de la sostenibilidad ambiental, temas muy bien abordados por el Doctor Antonio Elizalde Hevia de la Universidad Bolivariana de Chile en su artículo "¿Qué desarrollo es sostenible en el siglo XXI? Se revisan y se comentan diferentes puntos de vistas de lo que debería ser la sostenibilidad ambiental como un tema indispensable en la discusión de cualquier modelo de crecimiento económico que se pretenda establecer.

Son diversos los puntos de vistas que se exponen, desde los que opinan por un ilimitado crecimiento de la economía, aunque esto signifique devastar las últimas reservas de recursos naturales que le quedan a nuestro planeta, pasando por los que opinan que el desarrollo tecnológico y la ciencia, lo mismo que la gran empresa industrial son la tabla de salvación para los problemas de sostenibilidad ambiental de nuestro planeta, hasta llegar a los enfoques de la llamada "Ecología profunda" que aboga por un crecimiento económico que armonice con el equilibrio del medio ambiente ecológico del mundo que nos rodea (Kliksberg 2013).

Estas últimas propuestas propugnan por el respeto a los límites de la naturaleza, por un enfoque transdiciplinario de las propuestas de crecimiento y desarrollo económico y por una lógica de convivencia con la naturaleza con base en el "buen vivir" que permita al hombre la posibilidad de su desarrollo vinculándose con la naturaleza desde una posición de respeto.

\section{ReVisión de LA Literatura}

\section{Un abordaje desde la Economía Ambiental}

Recientemente he revisado un texto del Emérito profesor de la universidad de Massachusetts, Barry C. Field, escrito en el año 1995 bajo el título "Economía Ambiental, Una introducción”, me parece importante su esfuerzo por contribuir desde la óptica económica, a la solución de los problemas ambientales que aquejan hoy a nuestra sociedad.

Su pregunta ¿por qué las personas se comportan de manera tal que ocasionan daños al medio ambiente? (Field, 1995, p4) Plantea interrogantes muy importantes y mantiene abierto el debate sobre el comportamiento de las sociedades post modernas respecto al cuido y mantenimiento del medio ambiente natural que nos rodea.

En principio disentimos de él respecto a su percepción del exclusivo papel que se le asigna a la naturaleza como proveedor de materia prima e insumos de energía; no obstante, nos parece admirable su enfoque respecto a la necesidad del mantenimiento del equilibrio básico de los sistemas ecológico en que el hombre interviene para el "necesario" desarrollo de su actividad económica, sin embargo me parece que su preocupación radica no en lo que representa el deterioro del medio ambiente para la supervivencia de la especie humana sino más bien, en el agotamiento de las fuentes de recursos naturales indispensables,

\section{NotAS DE AUTOR}


como el mismo señala, para el desarrollo de los procesos productivos de los llamados países desarrollado o industrializados.

Señala Field en su libro la preocupación por el agotamiento de los recursos energéticos, especialmente de los que provienen de materiales fósiles como el carbón, el petróleo o el gas natural indispensables para el funcionamiento de la "gran industria petroquímica", observa además en su escrito, el deterioro que sufren los recursos minerales y forestales fuente indispensable de insumos en las denominadas "sociedades de la información" y no deja de mostrar preocupación por el deterioro de la calidad de los recursos hídricos y del aire "insumos vitales en casi todos los procesos de producción".

Es evidente el sesgo economicista del enfoque planteado por el autor de tan importante libro, sin embargo me parece interesante un modelo analítico que propone para el mantenimiento de lo que él llama el equilibrio fundamental relacionado a la producción y manejo de desechos, de diversas índoles, generados por el hombre, en su condición de productor y/o consumidor de bienes, en los procesos productivos.

En su modelo Field plantea que existen esencialmente tres formas de reducir las cantidades de materia prima $(\mathrm{M})$ y residuos $(\mathrm{R})$ descargados por el hombre en el medio ambiente:

- Primero plantea que es posible reducir las cantidades de residuos descargados a la naturaleza por efectos de las actividades antropogénicas disminuyendo las cantidades de bienes y servicios generados en la economía.

Dieciocho años después de haber sido expuesto ese enunciado, considero que tal planteamiento requiere de la atención de los responsables de la planificación económica no solo de las llamadas sociedades industriales sino también de los gobiernos y empresas de los países en vías de desarrollo.

- En segundo lugar plantea que otra forma de reducir $M$ es mediante la disminución de residuos provenientes de las industrias. Para ello propone dos formas alternativas para lograrlo: una de ellas es el invento y adopción de nuevas tecnologías y la otra la adopción de nuevas prácticas de producción.

La pregunta que flota en el aire a la luz de esta propuesta es ¿Existe voluntad de las grandes economías en sacrificar recursos económicos en pro del cuido del medio ambiente, poniendo en riesgo lo que ellos llaman "eficiencia económica"?

. Un último planteamiento del modelo propuesto es incrementar el "reciclaje" de los residuos provenientes de la producción y el consumo.

Al final considero que los tres planteamientos se supeditan o se podrían resumir en uno sólo: reducir el deseo consumista de nuestras sociedades, sobre todo de bienes y servicios que demandan grandes cantidades de recursos materiales y energéticos de la naturaleza.

Habiendo leído a este y otros autores me parece que existen planteamientos diversos, tanto como los puntos de vistas de cada uno de ellos, respecto a qué modelo de desarrollo nos permitirá aprovechar y preservar los ya casi agotados recursos naturales de manera tal que permitamos a las generaciones futuras gozar al menos de las potencialidades naturales que hoy nosotros disfrutamos.

Nos encontramos entonces en una encrucijada entre los enfoques de la Economía ordinaria cuyo paradigma principal es la "eficiencia de los procesos productivos", sin reparar en el agotamiento de los recursos naturales y los nuevos enfoques que plantean un crecimiento económico que armonice con las condiciones y las leyes del mundo físico natural.

En un mundo que cambia a un ritmo vertiginoso y que crece de manera irracional y desmesurada, dominado, este mundo, por enfoques globalizadores en el que los daños ambientales a nuestro planeta en los últimos cincuenta años han desmejorado las condiciones naturales de la tierra más que todos los sistemas históricos anteriores, los conceptos de ecológico y sostenible son hoy el centro de los discursos y retóricas de políticos y empresarios de las economías industriales; sin embargo todo queda en eso: discurso.

Los líderes de estas economías tratan de tranquilizar a los pobladores de sus países maquillando y disfrazando los verdaderos daños ocasionados al medio ambiente llamando a los consumidores a adoptar medidas de austeridad y de consumo responsable sin ellos haber cambiado antes sus formas de gestión y 
criterios de comportamiento los que al final, en nombre de la eficiencia económica, causan un enorme daño al medio ambiente ecológico no sólo de sus naciones sino del planeta tierra en general.

Fueron los Fisiócratas en el siglo XVIII encabezados por Quesnay y Turgot, entre otros, los que en un primer acercamiento a la Teoría económica plantearon la necesidad de un gobierno que armonizara el crecimiento económico con el progreso social, fundamentado su práctica en un modelo que permitiera el crecimiento físico y monetario de las naciones sin menoscabo del crecimiento físico de las riquezas naturales.

Tales planteamientos parecen haber quedado en el olvido y las nuevas corrientes económicas, sobre todas las que sustentan el modelo capitalista de producción, mantienen intacto los postulados de finales del siglo XIX e inicios del siglo XX que dan primacía a la práctica de "revender con beneficio"(Naredo,1996,p5).

El concepto de eficiencia económica domina hoy más que nunca los modelos de gestión económica en gran parte del mundo, con pequeñas excepciones en algunas naciones del norte de Europa y más recientemente en el sur de nuestra América donde existe una mayor racionalidad en el uso de los recursos naturales. En tal sentido, en un modelo sustentable el análisis de productividad ha sido inminente, los esfuerzos para desarrollarlo inician con (Farrell, 1957), este nuevo enfoque es conocido como análisis de datos envolventes y considera un enfoque no parcial de los factores productivos, otros autores mejoran el enfoque como (Färe R. y., 1978), (Färe R. S., 1994), and (Battese, \& Coelli, \& Ba, 1995.). De igual manera, encontramos otros autores que han desarrollado el modelo empíricamente aplicado a los diferentes sectores de la economía de América Latina (Bravo-Ureta \& \& Pinheiro, 1993), (Zúniga-González C. A., 2012), (Ludena, 2007).

Han surgido corrientes de pensamientos que abordan propuestas de desarrollo en el marco de lo que se ha dado por llamar "desarrollo sustentable" pero nuevamente surge la interrogante: ¿Qué desarrollo es sustentable en el siglo XXI?.

Parece interesante un primer planteamiento que propone abordar el problema de la sostenibilidad desde la perspectiva de la noción de límite: "Nada puede crecer indefinidamente”, esta posición se contrapone a los planteamientos del modelo capitalista de que los límites no existen, que las únicas fronteras que limitan al hombre son las auto impuestas, esto nos lleva a comprender entonces el comportamiento voraz de las grandes transnacionales de la industria y el comercio que han venido depredando los recursos naturales de nuestro planeta, basados en que cualquier límite en lo físico, lo ecológico o lo económico siempre podrá superarse con la tecnología aunque ésta cause profundos daños al medio ambiente natural donde se utiliza.

Un ejemplo de la arrogancia de los nuevos teóricos del capitalismo radica en su desprecio a los pensadores que consideran inviable e insostenible el ritmo de crecimiento de las sociedades industriales tal como lo señala Elizalde al citar un artículo de Morris Berman en su libro "El reencantamiento del mundo" donde afirma y señala que quienes defienden la tesis de que el crecimiento económico de las naciones solo es posible mediante el respeto de los límites físico de la naturaleza son "ocultistas", "místicos", "románticos pastorales" "ideólogos de estados estacionarios” etc.(Elizalde,2009,p57)

La crítica de Berman no repara en señalar ya no digamos en aceptar que "solamente el capitalismo histórico ha llegado a ser una amenaza para la posibilidad de una existencia futura viable de la humanidad, por haber sido el primer sistema histórico que ha englobado toda la Tierra y que ha expandido la producción y la población más allá de todo lo previamente imaginable (Wallerstein, 2008).

A todos estos "ocultistas" y "místicos" "masa amorfa de ecólogos" "hombres de pensamientos arcaicos" como llama Morris Berman los respalda una afirmación hecha por el mismo Immanuel Wallerstein, quien citado por Elizalde asegura:

Los dilemas ambientales que encaramos hoy son resultado directo de la economía del mundo capitalista. Mientras que todos los sistemas históricos anteriores transformaron la ecología, y algunos de ellos llegaron a destruir la posibilidad de mantener en áreas determinadas un equilibrio viable que asegurase la supervivencia del sistema histórico localmente existente, solamente el capitalismo histórico ha llegado a ser una amenaza para la posibilidad de una existencia futura viable de la humanidad, por haber sido el primer sistema histórico 
que ha englobado toda la Tierra y que ha expandido la producción y la población más allá de todo lo previamente imaginable.

Es inverosímil: de todos los recursos extraídos por el hombre de la tierra la mitad fueron agotados en todos los miles de años anteriores a 1922 la otra mitad fue agotada en solamente 70 años hasta 1992) 21 años después ¿cuál será la tasa de agotamiento de los recursos naturales en la actualidad?

Un debate importante que plantea Arne Naess, es la distinción entre Ecología superficial y Ecología profunda, afirmando que la primera de éstas ataca la contaminación y el agotamiento de los recursos naturales sin identificar y señalar las causas políticas, sociales y culturales de la crisis ambiental que provocan. (Elizalde, 2008, p.58)

Se observa interesante el planteamiento que hace Naess para confrontar el limitado enfoque de la ecología superficial respectos a las crisis ambientales actuales: Introduce el concepto de "Ecología profunda" asegurando que ésta si estudia las causas de los males ambientales que aquejan a nuestro planeta haciendo una fuerte crítica a los sistemas de vida y al conjunto de valores éticos y morales de las sociedades industriales, además señala la importancia de un enfoque más filosófico para el abordaje de la problemática ambiental actual.

Observamos otros enfoques que proponen modelos de desarrollo sustentable, los cuales se revisan a continuación:

\section{. El "negacionismo" capitalista}

Los comentarios de Jorge Riechman, citado por Elizalde, llaman poderosamente la atención pues lleva a otro plano el análisis de los problemas ambientales y lo aterriza en lo que él denominó "Las huidas", una especie de escapismos que propone el hombre frente a la dificultad de resolver los problemas de deterioro de nuestra naturaleza, al punto tal que, en su incapacidad de responder a las reacciones de la naturaleza, una vez que se han traspasado sus límites, propone las colonización interplanetaria, entre otras propuestas tales como la fusión nuclear y la nano tecnología como una salida a los límites del crecimiento económico. Frente a este y otros planteamientos retrógrados nuevamente resurge la propuesta "Ecológica" de respeto a los límites físicos de la naturaleza.

Fue en el año 1972 que el enfoque de "Los límites del crecimiento" surge como una advertencia de que si el actual incremento de la población mundial, la industrialización, la contaminación, la producción de alimentos y la explotación de los recursos naturales se mantiene sin variación, alcanzará los límites absolutos de crecimiento en la Tierra durante los próximos cien años.

Pasado 40 años del surgimiento de este enfoque, en el año 2012, se publicó el libro Les limites à la croissance, en el que se asegura que ya estamos en los límites.

\section{- Del capitalismo verde y la desmaterialización.}

La desmaterialización es un enfoque ya abordado en este escrito, de él hace referencia Barry C. Field en su libro Economía Ambiental, aunque lo importante del tema es la crítica que se hace a la propuesta desmaterializadora en la paradoja de Jevons, de que tal propuesta al reducir los costos de producción, con adopción de nuevas tecnología solamente provocará un mayor consumo de bienes.

Del capitalismo verde quiero hacer referencia, observando el desempeño de la industria licorera en nuestro país (Nicaragua) y el papel de la gran industria privada como la tabla de salvación para la preservación de la naturaleza, como propone Francés Cairncross (1993; 1996), redactora jefe de The Economist. Por un lado promueven programas y proyectos verdes con la adopción de tecnologías de punta que les permite producir energía limpia, pero por otro lado promueven a todos los niveles, haciendo usos de los medios de comunicación en su poder, el consumo de bebidas alcohólicas, causa principal de muchos males sociales tales como los accidentes de tránsito, la destrucción de hogares y familias y las muertes de los más vulnerables como consecuencia del consumo de cantidades excesivas de los productos que ellos elaboran y promocionan. Así veo yo la propuesta de "Capitalismo Verde": La acumulación de capital sin reparar en los daños sociales que en este proceso se provocan. 


\section{- Nuevas corrientes ecológicas: Las utopías que sobreviven}

Se apuntan en estos nuevos enfoques los críticos al desarrollismo y al crecimiento desmedido propuesto y practicado por las grandes economías industriales y a sus ideólogos que sostienen, tal como lo hace Berman, en su libro El Reencantamiento del Mundo:

Que el mejor de los caminos es el que retiene todas las suposiciones de la Revolución Industrial y nos llevaría hacia la salvación a través de la ciencia y la tecnología; Sus proponentes visualizan una economía expansiva, mayor urbanización y homogeneidad cultural siguiendo el modelo occidental como algo bueno e inevitable (Elizalde,2009,p57)

Revisamos entonces los planteamientos de "El decrecimiento", "La biomímesis” y el "Sumak Kawsay", enfoque todos un tanto utópicos pero dignos de ser considerados.

El primero de estos planteamientos propone un cambio radical de los hábitos de vida hasta ahora equivocados, basados en modelos de sociedades de consumo en el que el objetivo fundamental es crecer por crecer sin considerar los daños ambientales que al mundo natural provoca ese incesante afán de acumulación y riqueza de los dueños del capital.

La propuesta resalta la necesidad de cambiar una agricultura transgénica por una agricultura ecológica, un vivir marcado por el individualismo por uno que propugne por el bienestar de la colectividad, la desurbanización y desindustrialización, la des globalización de la economía por una producción a escala local, en que se potencien las capacidades locales a través de la gestión comunitaria.

La Biomímesis: un término usado en la ciencia y en la ingeniería para definir a la naturaleza como fuente de inspiración y para hacer referencia al proceso de entender y aplicar a problemas humanos, soluciones procedentes de la naturaleza en forma de principios biológicos, biomateriales, o de cualquier otra índole. Una vez más se presenta un enfoque de sostenibilidad relativo al respeto de los límites naturales y a la necesaria armonía que deben guardar las propuestas de crecimiento económico con el mundo natural que nos rodea.

El enfoque presenta una propuesta de mejoramiento de la calidad de la vida humana, sin tratar de aventajar a la naturaleza sino más bien imitarla. Encontramos muchos ejemplos en la naturaleza que deberán servirnos como referentes necesarios al momento de dictar políticas o definir modos de actuar en la incesante tarea de crear condiciones óptimas que permitan la sobre vivencia de la especie humana tales como la forma de filtrar el agua, limpiar el aire o nutrir el suelo.

Una propuesta interesante que nos obliga a revisar nuestra relación con la naturaleza y definir el “entramado de relaciones que nos permita la reconstrucción ecológica de la economía” sustentado en un transformación de lo urbano, lo industrial y lo agrario en modelos lo más parecidos a la naturaleza.

El Sumak Kawsay o el buen vivir, modelo ancestral de las culturas originarias del sur de América. Cuando revisé el significado de la frase, el primero de los vocablos "Sumak" hace referencia a la "realización ideal y hermosa del planeta", el segundo de ellos a la "vida en plenitud". Cuanta Utopía, pero muy necesaria para mantener nuestros sueños de un mundo más digno de ser heredado a nuestros hijos.

Se cierra este breve análisis revisando un poco algunos postulados finales respecto a modelos alternativos de desarrollo entre los que destacan "La ética del consumo" y "El principio del abajamiento".

La ética del consumo señala la urgente necesidad de cambiar los hábitos de consumo no sólo de nuestras sociedades en su conjunto sino a partir del ámbito personal, de nosotros como persona, de apartarnos un poco de la exterioridad y volvernos en busca de la dimensión interna.

Su tesis principal se fundamenta en el enunciado de «no consumas nunca productos cuyo consumo no se puede universalizar sin producir daño a las personas o al medio ambiente». Es un llamado urgente a no seguir en la lógica consumista que nos han y que nos hemos auto impuesto y que "obliga" a la gran industria a seguir depredando las ya casi agotadas reservas de recursos naturales.

Por otro lado la tesis de "El abajamiento" sostiene que "es la solidaridad que se despliega en la piedad ante el otro, en el reconocimiento del otro y en la universalidad para el otro, la energía vital y el paradigma a partir del cual nuestra sociedad puede tomar conciencia de sí misma y buscar con urgencia soluciones”. 
Me parece la más transdisciplinaria de las propuestas, un enfoque humanista que recurre al llamado de la conciencia humana como la alternativa más viable para enfrentar la "actual crisis civilizatoria".

- Incorporación de aspectos ambientales en los modelos de desarrollo

En América Latina hemos notado un esfuerzo por incorporan en el modelo de desarrollo aspectos medio ambientales desde el enfoque de Análisis de Datos Envolventes DEA (Dios-Palomares, y otros, 2015), (Zúniga-González, Toruño, \& Navas-Calderon, 2015), (Zúniga-González, , Blanco-Roa,, Berrios, MartínezAvendaño, \& Navas-Calderon, 2015). En estos modelos los autores incluyen con un enfoque DEA un componente ambiental, es decir consideran que el modelo económico es influenciado por variables medio ambientales, en condiciones de variaciones climáticas.

\section{- La Bioeconomía y el Cambio Climático}

En un contexto donde el ritmo de crecimiento poblacional es exponencial, la variación climática es inevitable y la demanda de mayores recursos en un modelo de desarrollo sustentable ha originado una nueva epistemología de la Economía Biobasada (Maldonado, 2012), (Mohammadian, 1999), (ZúnigaGonzález, , y otros, 2014). En un modelo de desarrollo rural sustentable es importante considerar la tendencia mundial de las actividades económica donde las unidades de producción se identifican con los senderos de la bioeconomia: Explotación de los recursos de la biodiversidad, Eco intensificación, Biorefienria / Bioproductos, Aplicaciones de la Biotecnología, Eficiencia Energética, Valor agregado en la cadena de valor (Zúniga-González, , y otros, 2014,.Zuniga et al., 2014) Las economías de los países, al menos de Iberoamérica están adoptando medidas a las variaciones del cambio climático y por tanto el modelo de desarrollo rural sustentable de manera multidisciplinar integra variables ambientales y está en estrecha relación con las variables sociales, políticas, productivas, y de gobernanza (Urbina 1994, Zuniga et al., 2014).

\section{Conclusiones}

Quizás la interrogante planteada por Field de ¿por qué las personas se comportan de manera tal que ocasionan daños al medio ambiente? No sea respondida de manera clara durante mucho tiempo, sin embargo los esfuerzos actuales por transformar la cultura humana, hasta hoy dominada por el afán de enriquecimiento desmedido y el consumismo, son válidos.

El surgimiento de una propuesta enmarcada en lo que se denomina "Ecología Profunda" es un planteamiento interesante a ser tomado en cuenta por los diseñadores de políticas nacionales, por los teóricos de la economía, por los ambientalistas y por todas las personas relacionadas o no con la imperiosa necesidad del restablecimiento o al menos preservación de las condiciones naturales del mundo externo que nos rodea. Complementa este enfoque la nueva epistemología de la Bioeconomia y el Cambio Climático que aborda los senderos productivo de la bio economía y nuevo instrumento para medir la nueva Economía Bio basada.

\section{Referencias Bibliografía}

Altieri, M. \&. (2000). Teoría y práctica para una agricultura sustentable. Serie Textos Básicos para la Formación Ambiental. PNUMA. Red de Formación Ambiental para América Latina y el Caribe. México, 235.

Artaraz, M. (2002). Teoría de las tres dimensiones de desarrollo sostenible. Ecosistemas, 11(2).

Battese, G. E., \& Coelli, T. J. (1995.). A model for technical inefficiency effects in a stochastic frontier production function for panel data. Empirical economics, 20(2), 325-332.

Bravo-Ureta, B. E., \& Pinheiro, A. E. ( 1993). Efficiency analysis of developing country agriculture: a review of the frontier function literature. Agricultural and resource economics Review, 22(1), 88-101.

Dios-Palomares, R., Alcaide, D., Diz, J., Jurado, M., Prieto, A., Morantes, M., \& Zuniga-Gonzalez, C. A. (2015). Análisis de la eficiencia de sistemas agropecuarios en América latina y el Caribe mediante la incorporación de aspectos ambientales. Revista Científica Veterinaria, 25 (1), 43-50. 
Elizalde, A. (2009). ¿Que desarrollo puede llamarse sostenible en el siglo XXI? La cuestión de los límites y las necesidades humanas. Buenos Aires: Revista Educación. p 53-75.

Färe, R. S. (1994). Productivity growth, technical progress, and technical change in industrilized countries. American Economic Review 84, pp 66-83.

Färe, R. Y. (1978). Measuremnet Technical efficiency of production. Journal of Economic theory, 19, pp 150-162

Farrell, M. (1957). Measurement of productive efficiency. Journal of the Royal Society, Serie A, 120 (3), 253-581.

Field, B. C. (1995). Economía Ambiental. Bogotá: Mc. Graw Hill.

Garza, E. G. (2008 ). De las teorías del desarrollo al desarrollo sustentable: historia de la constitución de un enfoque multidisciplinario. Ingenierías, 11(39), 4.

Kliksberg, B. (2013). Etica para empresarios. Buenos Aires: Ediciones Ética y Economía.

Ludena, C. E. (2007). "Productivity Growth and Convergence in Crop, Ruminant and Non- Ruminant Production:. Agricultural Economics , 37: 1-17.

Maldonado, C. (2012). Bioeconomía-Economía ecológica. Desde abajo, 16-19

Mohammadian, P. D. (1999). Bioeconomía: nuevo paradigma para la problemática medioambiental. Observatorio medioambiental, (2), 41-56.

Schejtman, A. (2004). Desarrollo territorial rural. . Debates y temas rurales, 1, 7-46

Urbina, G. B. (1994). Evaluación de proyectos. Mexico D.F: Mc. Graw Hill.

Zúniga-González, , C. A., Blanco-Roa,, N. E., Berrios, R., Martínez-Avendaño, J., \& Navas-Calderon, J. (2015). Impacto de la reducción de Metano en las Economías Verde de los sistemas de producción pecuaria de América Latina. Universitas (León). Revista Cien, 6(1), 30-48.

Zúniga-González, C., Dios-Palomares, R., Rangel Cura, , R., Aguilar, A., Blanco Roa, N., Toruño, P. \& Caballero Hernández, A. (2014). La Bioeconomía: Una nueva perspectiva de la Economía. En R. d. Climàtico, Estado del Arte de la Bioeconomia y el Cambio Climático (pág. 21). Nicaragua: Editorial Universitaria UNAN-León.

Zúniga-González, C., Sardiñas Gómez, J., O., Quiros, O., Blanco Roa, , N., Colón García,, A., \& Rangel Cura, , R. (2014). Los senderos productivos de la bioeconomía: Una revisión en ALC. En R. d. Climático, Estado del arte de la bioeconomia y el cambio climático (pág. 2.2). Nicaragua: Editorial Universitaria UNAN-León.

Zúniga-González, C. A. ( 2012). Total Factor Productivity and the Bio Economy Effects. Journal of Agricultural Studies, , 1(1), 1-29.

Zúniga-González, C. A., Toruño, P. J., \& Navas-Calderon, J. (2015). Impacto de los sistemas forestales en la Economía Verde con énfasis en la producción pecuaria de Centro América y el Caribe. Universitas (León). Revista Científica de la UNAN-León, 6(1), 49-69

Field, B. C. (1997). Economía ambiental.

Naredo E, Moller I, Moragues C, de Agustin JJ, Scheel AK, Grassi W, et al. Interobserver reliability in musculoskeletal ultrasonography: results from a "Teach the Teachers" rheumatologist course. Ann Rheum Dis 2006;65:14 -9.

Wallerstein, I. (2008). L'universalisme europeu: La retòrica del poder (Vol. 19). Universitat de València.

cairncross, F. (1993). Las cuentas de la Tierra: economía verde y rentabilidad medioambiental. Madrid: Acento Editorial.

cairncross, F.(1996). Ecología S.A. Hacer negocios respetando el medio ambiente. Madrid: EcoEspaña. 